OPEN ACCESS

Edited by:

Catherine Proenza,

University of Colorado Denver,

United States

Reviewed by:

Arie O. Verkerk,

University of Amsterdam, Netherlands Andrew F. James,

University of Bristol, United Kingdom

T Alexander Quinn,

Dalhousie University, Canada

*Correspondence:

David J. Christin

dchristi@med.cornell.edu

Specialty section:

This article was submitted to

Cardiac Electrophysiology,

a section of the journal

Frontiers in Physiology

Received: 01 September 2017 Accepted: 13 December 2017

Published: 04 January 2018

Citation:

Ortega FA, Grandi E, Krogh-Madsen T and Christini DJ (2018) Applications of Dynamic Clamp to Cardiac Arrhythmia Research: Role in Drug Target Discovery and Safety Pharmacology Testing. Front. Physiol. 8:1099. doi: 10.3389/fphys.2017.01099

\section{Applications of Dynamic Clamp to Cardiac Arrhythmia Research: Role in Drug Target Discovery and Safety Pharmacology Testing}

\author{
Francis A. Ortega ${ }^{1}$, Eleonora Grandi ${ }^{2}$, Trine Krogh-Madsen ${ }^{3}$ and David J. Christini ${ }^{1,3 *}$ \\ ${ }^{1}$ Physiology, Biophysics, and Systems Biology Graduate Program, Weill Cornell Graduate School of Medical Sciences, \\ New York, NY, United States, ${ }^{2}$ Department of Pharmacology, University of California, Davis, Davis, CA, United States, \\ ${ }^{3}$ Greenberg Division of Cardiology, Weill Cornell Medical College, New York, NY, United States
}

Dynamic clamp, a hybrid-computational-experimental technique that has been used to elucidate ionic mechanisms underlying cardiac electrophysiology, is emerging as a promising tool in the discovery of potential anti-arrhythmic targets and in pharmacological safety testing. Through the injection of computationally simulated conductances into isolated cardiomyocytes in a real-time continuous loop, dynamic clamp has greatly expanded the capabilities of patch clamp outside traditional static voltage and current protocols. Recent applications include fine manipulation of injected artificial conductances to identify promising drug targets in the prevention of arrhythmia and the direct testing of model-based hypotheses. Furthermore, dynamic clamp has been used to enhance existing experimental models by addressing their intrinsic limitations, which increased predictive power in identifying pro-arrhythmic pharmacological compounds. Here, we review the recent advances of the dynamic clamp technique in cardiac electrophysiology with a focus on its future role in the development of safety testing and discovery of anti-arrhythmic drugs.

Keywords: dynamic clamp, cardiac electrophysiology, cardiac modeling, arrhythmia mechanisms, antiarrhythmic drugs, pharmacology \& drug discovery

\section{INTRODUCTION}

The search for successful anti-arrhythmia therapeutics is rooted in the voltage clamp and current clamp techniques, which have provided the mechanistic details behind the ionic membrane currents that compose the cardiac action potential (AP). While basic science has made great leaps in identifying and characterizing the basic factors involved in arrhythmia, the translation of these advances into successful therapies has been lackluster. Nonetheless, investigators have been using a combination of experimental and computational approaches to unravel the complex mechanisms underlying cardiac arrhythmia. Using this approach, experimental measurements, typically in single cells from mammalian hearts, are used to develop biophysically detailed mathematical models that can be scaled up to the tissue and whole-organ levels where arrhythmia occurs. Unlike experiments, computational modeling readily allows for the precise perturbation of particular parameters individually or in controlled combinations (simulating, e.g., the multifactorial nature of many disorders), but results are reliant on the accuracy of the model and its many components. The dynamic clamp technique is a merger between experimental and computational techniques that has been gaining traction as a hybrid method for elucidating 
arrhythmia mechanisms and possible therapeutics.

Traditional patch clamp protocols are typically static and predetermined, such as sequential voltage steps used to study membrane current dependencies. Dynamic clamp is an extension of patch clamp, where measurements from the cell are used to modify a continuously changing experimental protocol in a real-time feedback loop (Robinson and Kawai, 1993; Sharp et al., 1993). Earlier work has shown broad application-coupling of separate cardiomyocytes through an artificial gap junction (Tan and Joyner, 1990; Joyner et al., 1991; Spitzer et al., 1997; Verheijck et al., 1998; Zaniboni et al., 2000; Huelsing et al., 2001), injection of measured current from a transfected cell into a primary isolated myocyte (Berecki et al., 2005, 2006), antrhomorphization of mouse cardiac APs (Ahrens-Nicklas and Christini, 2009; Bot et al., 2012), and more recently in the study of cardiomyocyte coupling to unexcitable cells (McSpadden et al., 2012) and fibroblasts/myofibroblasts (Nguyen et al., 2012; Brown et al., 2016). The history of dynamic clamp has been reviewed in detail elsewhere (Prinz et al., 2004; Wilders, 2006; Ravagli et al., 2016). Here, we focus on a specific configuration of this technique, called the dynamic model clamp (referred hereafter as dynamic clamp), where a mathematically based model of a conductance is injected to the cell in real-time. Characteristically, this mathematical model describes a specific voltage and time-dependent membrane current determined by a set of differential equations. Measured voltage of a cell in a patch clamp configuration is fed into a mathematical model at high rates, from which the calculated current is injected back into the cell (Figure 1A).

Central to the dynamic clamp experimental rig is the software, which acts as the interface between the patch-clamp hardware and mathematical models. Accurate and rapid sampling of the membrane potential and computation of the virtual conductance is required to mimic sufficiently a biological conductance (Bettencourt et al., 2008). These requirements necessitate hard real-time control. In this context, the feedback loop must complete every iteration within a specified time constraint, typically $50-100 \mu \mathrm{s}(10-20 \mathrm{kHz})$ in cardiomyocyte dynamic clamp experiments, a feat not possible on standard operating systems and software due to technical limitations. The works discussed here predominately use two software platformsDynaClamp (Berecki et al., 2005, 2006) and the Real-Time eXperimental Interface (RTXI, www.rtxi.org; Ortega et al., 2014; Patel et al., 2017). Both platforms utilize a customized real-time Linux operating system and are freely available.

In this review, we discuss how investigators have used the dynamic clamp technique to test theoretical drug targets, validate and improve existing cardiac mathematical models, and design assays for cardiotoxicity testing.

\section{INVESTIGATION OF ARRHYTHMIA MECHANISMS}

\section{Drug Target Identification}

Dynamic clamp studies on the cardiac L-type $\mathrm{Ca}^{2+}$ current $\left(\mathrm{I}_{\mathrm{CaL}}\right)$ by Madhvani et al. identified arrhythmia mechanisms, which could potentially be targeted by anti-arrhythmic drugs (Madhvani et al., 2011, 2015). The authors specifically focused on the role of $\mathrm{I}_{\mathrm{CaL}}$ in the formation of early after depolarizations (EADs), i.e., secondary depolarizations during phase 2 and 3 of the AP resulting from a transient failure of AP repolarization. EADs are used as a marker of cardiac arrhythmia due to its propensity to trigger a premature AP and subsequently initiate cardiac arrhythmias, such as Torsades de pointes (TdP) or ventricular fibrillation, which in turn can lead to sudden cardiac death (Cranefield and Aronson, 1991). EADs require an inward current that can overcome and reverse repolarization, which can be fulfilled by $\mathrm{I}_{\mathrm{CaL}}$, the major inward current during phase 2 and 3 of the AP. Madhvani et al. aimed to investigate the dependence of EADs on the biophysical properties of $\mathrm{I}_{\mathrm{CaL}}$, but the lack of an assortment of drugs known to finely alter this current makes traditional patch clamp experiments impractical. Thus, to mimic theoretical perturbations to $\mathrm{I}_{\mathrm{CaL}}$ properties in vitro dynamic clamp was used instead.

In rabbit ventricular myocyte exhibiting EADs, induced with either hydrogen peroxide (Figure 1B, top) or hypokalemia, they replaced native $\mathrm{I}_{\mathrm{CaL}}$ (blocked with nifedipine) with a virtual model-based $\mathrm{I}_{\mathrm{CaL}}$, which was injected using dynamic clamp (Figure 1B, middle). The consequences of alterations in $\mathrm{I}_{\mathrm{CaL}}$ biophysical properties were investigated by manipulating the parameters underlying the modeled current. For example, shifting the half-maximal activation voltage by $5 \mathrm{mV}$ abolished EADs and returned AP duration (APD) to normal values (Figure 1B, bottom). Note that $\mathrm{H}_{2} \mathrm{O}_{2}$ affects multiple inward currents in addition to $\mathrm{I}_{\mathrm{CaL}}$, such as the late sodium current (Xie et al., 2009), but modification of $\mathrm{I}_{\mathrm{CaL}}$ alone was able to eliminate EADs.

The mechanistic basis for the observed behavior was established in earlier work describing a window current region between -40 and $0 \mathrm{mV}$ (January and Riddle, 1989) where the steady-state activation and inactivation curves overlap. In this region, a fraction of the L-type $\mathrm{Ca}^{2+}$ channels are not inactivated and available for possible reactivation and generation of an EAD. A positive shift in the steady-state activation curve reduces this window region and eliminates EADs. In their later work, Madhvani et al. systematically perturbed all $\mathrm{I}_{\mathrm{CaL}}$ model parameters and measured the consequences to EAD formation, confirming that parameter changes that reduced the window current region (depolarizing shifts to steady-state activation, or hyperpolarizing shifts to steady-state inactivation) were highly effective at EAD prevention (Madhvani et al., 2015). Based on these observations, the authors identified the purine analog Roscovitine, originally developed as an anti-cancer agent, as a promising anti-arrhythmic due to its ability to decrease the window current through a reduction to the late component of $\mathrm{I}_{\mathrm{CaL}}$. Preliminary work has shown Roscovitine did indeed abolish EADs in myocytes and terminated ventricular tachycardia/fibrillation in whole rat hearts (Karagueuzian et al., 2017), supporting its therapeutic potential. Notably, this work illustrates a new paradigm in the search for new classes of anti-arrhythmic drugs.

Using a similar approach to the $\mathrm{I}_{\mathrm{CaL}}$ studies, Altomare et al. investigated the human ether-a-go-go related gene (hERG) 


\section{A}

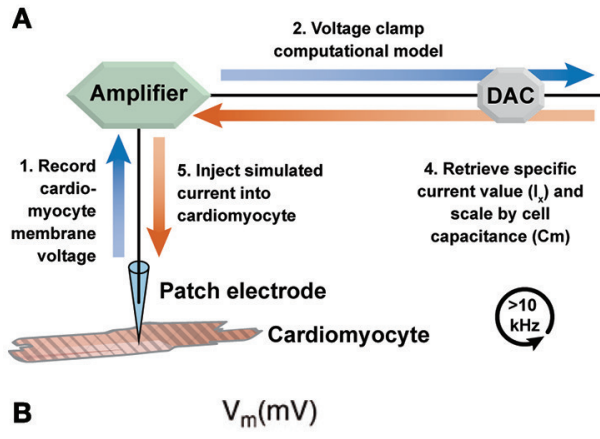

B
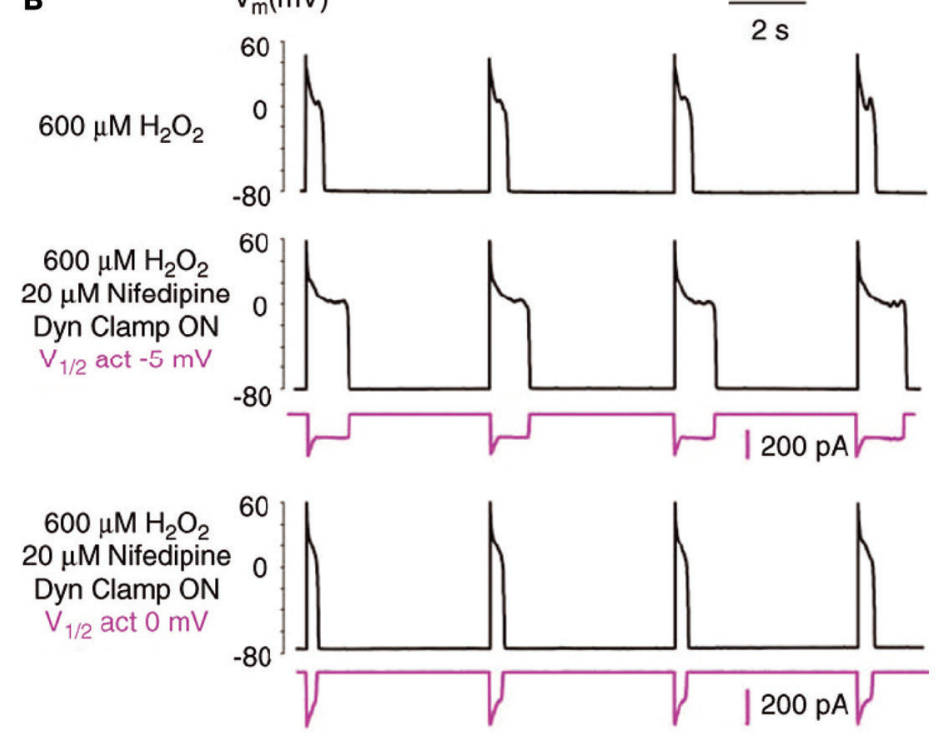

c

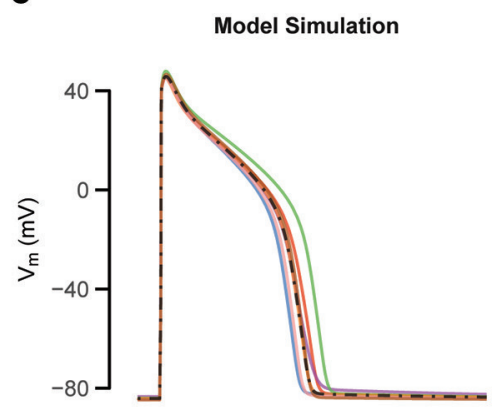

Dynamic Clamp Experiment

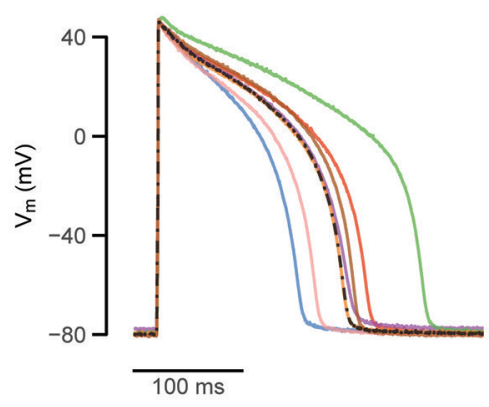

Current applied:

$-{ }^{-} I_{\mathrm{Kr}}-{ }^{-} \mathrm{I}_{\mathrm{K} 1}-{ }^{-I_{\mathrm{CaT}}-}-\mathrm{I}_{\mathrm{NCX}}$
$-+\mathrm{I}_{\mathrm{Ks}}-+\mathrm{I}_{\mathrm{CaL}}-+\mathrm{I}_{\mathrm{NaK}} \cdot \cdot$ Baseline

FIGURE 1 | Using dynamic patch clamp to reveal drug targets and systematically test computational models. (A) Schematic of the dynamic model clamp configuration. (B) Rabbit ventricular myocytes produce EADs during hydrogen peroxide exposure at a pacing cycle length of $5 \mathrm{~s}$ at $37^{\circ} \mathrm{C}$ (middle). Replacement of ${ }^{I} \mathrm{CaL}_{\mathrm{L}}$ with a virtual conductance through dynamic clamp after block with nifedipine recapitulates appearance of EADs (middle). By varying the half-maximal of activation by $5 \mathrm{mV}$, EADs are abolished (bottom). Adapted with permission (Madhvani et al., 2011). (C) Prediction of a $40 \%$ increase or decrease of different cardiac currents based on a computational model of a ventricular guinea pig cardiomyocyte are tested with dynamic clamp, revealing a substantial mismatch. Adapted with permission (Devenyi et al., 2017).

channel responsible for the rapid portion of the delayed rectifier $\mathrm{K}^{+}$current $\left(\mathrm{I}_{\mathrm{Kr}}\right)$ (Altomare et al., 2015). Mutations and drug perturbations to $\mathrm{I}_{\mathrm{Kr}}$ result in abnormal repolarization, clinically highlighted by long- or short- QT syndrome. The authors examined how $\mathrm{I}_{\mathrm{Kr}}$ biophysical properties influenced APD and its temporal variability by blocking and subsequently replacing native $\mathrm{I}_{\mathrm{Kr}}$ in guinea pig ventricular cardiomyocytes using dynamic clamp. The modeled current was shown to recover control AP parameters adequately, which reveals the properties described in the model are sufficient to describe the contribution of $\mathrm{I}_{\mathrm{Kr}}$ to APD and its stability. The voltage and time dependent properties of $\mathrm{I}_{\mathrm{Kr}}$ were systematically perturbed, and then compared to control and drug block conditions. This approach allowed a detailed examination of the consequences of each current property in isolation. The study showed both APD and its variability were most sensitive to changes to steadystate inactivation. Alternatively, while steady-state activation had little impact on APD, significant changes to APD variability were observed. This suggests that variability in APD, rather than mean APD, may be more sensitive in detecting $\mathrm{I}_{\mathrm{Kr}}$-dependent repolarization abnormalities.

Dynamic clamp has also been used successfully in studies of the transient outward $\mathrm{K}^{+}$current $\left(\mathrm{I}_{\text {to }}\right)$, where dynamic clamp was used to vary $\mathrm{I}_{\text {to }}$ conductance in ventricular (Dong et al., 2006, 2010; Nguyen et al., 2015) and atrial cardiomyocytes (Workman et al., 2012). Given the fact existing $I_{\text {to }}$ blocking drugs are nonselective (Ridley et al., 2003; Aréchiga-Figueroa et al., 2010), these studies provided important insight into the relationship between $I_{\text {to }}$ and the morphology and duration of the AP. Dong et al. sought to understand the impact of $I_{\text {to }}$ on the mechanical properties of cardiomyocytes. $I_{\text {to }}$ is responsible for the presence of the characteristic phase- 1 notch of the AP, and conflicting evidence suggested notch prominence can either increase or decrease $\mathrm{I}_{\mathrm{CaL}}$, respectively, enhancing or reducing contraction. Canine ventricular epicardial myocytes are characterized by a prominent phase-1 notch, which endocardial myocytes generally lack (Antzelevitch et al., 1991). By swapping I to conductance levels of both cell-types using dynamic clamp, Dong et al. found 
that endocardial cells in which the small native $\mathrm{I}_{\text {to }}$ was substituted by a larger epicardial-like $\mathrm{I}_{\text {to }}$ displayed diminished contractility, and demonstrated that $I_{\text {to }}$ acts as a negative regulator of contractility through reduction of $\mathrm{I}_{\mathrm{CaL}}$ peak magnitude (Dong et al., 2010).

Workman et al. investigated the influence of $\mathrm{I}_{\text {to }}$ on atrial arrhythmogenesis, a topic which was unclear due to the lack of $\mathrm{I}_{\text {to }}$ specific drugs (Workman et al., 2012). Reduction of $\mathrm{I}_{\text {to }}$ through dynamic clamp revealed AP prolongation, and additional $\beta$-adrenergic stimulation evoked EADs. $\mathrm{I}_{\text {to }}$ increase or exposure to the $\beta$-blocker atenolol prevented EAD formation. This suggests $\mathrm{I}_{\text {to }}$ enhancement holds promise in arrhythmia prevention, at least in the atrium. On the other hand, the dynamic clamp study by Nguyen et al. showed that $I_{\text {to }}$ enhancement potentiated EADs in rabbit ventricular myocytes with reduced repolarization reserve, i.e., the intrinsic redundancy against excessive APD (Roden, 1998). By affecting the early AP phases, $I_{\text {to }}$ augmentation can alter other voltage-dependent repolarization currents, leading to decreased late repolarization reserve and increased EAD formation (Nguyen et al., 2015).

It is important to note that the dynamic clamp technique suffers from a major limitation, i.e., the lack of ion selectivity in the current injection. Given physiological intracellular solutions contain predominantly $\mathrm{K}^{+}$, dynamic clamp of $\mathrm{I}_{\mathrm{CaL}}$ current will be carried mainly by $\mathrm{K}^{+}$, and not $\mathrm{Ca}^{2+}$. Thus, the simulated conductance-which should be $\mathrm{Ca}^{2+}$-dependent per se, is unable to trigger secondary intracellular $\mathrm{Ca}^{2+}$ release and contraction. In an attempt to compensate for this limitation, Madhvani et al. simulated the intracellular $\mathrm{Ca}^{2+}$ transient, which was then fed back into the $\mathrm{I}_{\mathrm{CaL}}$ model (Madhvani et al., 2011, 2015), whereas Devenyi et al. included ion selectivity in their simulations (Devenyi et al., 2017). While especially true for $\mathrm{Ca}^{2+}$ due to its major role as a secondary messenger, caution should be applied when interpreting results of virtual conductance injection, as transient changes in intracellular concentrations can affect ion channel behavior.

\section{Improvement of Cardiac Computational Models}

The Comprehensive in vitro Proarrhythmia Assay (CiPA) initiative seeks to introduce a new cardiac drug safety testing paradigm that combines in vitro drug effects on multiple ion channels, computational modeling of cardiac currents and AP, and the use of human stem-cell derived cardiomyocytes (Sager et al., 2014; Colatsky et al., 2016). Computational modeling has proven to be a vital tool in cardiac arrhythmia research, and is expected to be instrumental in the future pipeline in drug testing. Confidence in model accuracy is directly tied to dynamic clamp results, as errors in the formulation of the mathematical model used can skew results. However, this limitation can be exploited because only accurate models can fully rescue behavior after drug block.

Ravagli et al. compared two computational models of the hyperpolarization-activated funny current, $\mathrm{I}_{\mathrm{f}}$ (Ravagli et al., 2016), which plays a major role in the pacemaker activity current of sinoatrial node (SAN) cells. The authors used a dynamic clamp rescue experiment, where ivabradine was used to partially block $\mathrm{I}_{\mathrm{f}}$ current, and a dynamic clamp injected model current was used to rescue control behavior. They showed one model significantly outperformed the other by restoring spontaneous activity in SAN cells, identifying the more accurate mathematical formulation of their experimental data. Bartolucci et al. used this strategy to validate an optimized formulation of the $\mathrm{I}_{\mathrm{Kr}}$ current (Bartolucci et al., 2015). The original LuoRudy model (Luo and Rudy, 1994), derived from voltage clamp step protocols (Sanguinetti and Jurkiewicz, 1990), fit poorly to their experimentally measured $\mathrm{I}_{\mathrm{Kr}}$ current data obtained with AP clamp. After optimization to the AP clamp data, their new model strongly diverged from the widely used LuoRudy formulation and fully reversed $\mathrm{I}_{\mathrm{Kr}}$ block during dynamic clamp.

Devenyi et al. used dynamic clamp to artificially scale multiple cardiac currents in guinea pig ventricular myocytes using a single whole cell model (Devenyi et al., 2017). Altogether, this amounted to a rapid and efficient testing of multiple computationally-based hypotheses within the same cell under static conditions. By comparing their experimental results of the current perturbations to the predicted results from the computational model, the authors noted significant discrepancies (Figure 1C). First, the basal APD was shorter, and second, current perturbations in the experiment were generally larger than predicted by the model. The authors then used the new experimental data to reparameterize the model through unbiased fitting with a genetic algorithm, yielding a new model that could recapitulate the experimental data well. Interestingly, while the original model had a large ratio between the slow $\left(\mathrm{I}_{\mathrm{Ks}}\right)$ and rapid $\left(\mathrm{I}_{\mathrm{Kr}}\right)$ portions of the delayed rectifier $\mathrm{K}^{+}$current, the fitting consistently reversed this ratio. This finding was then verified experimentally, and further in-silico investigation into the consequences to cardiac arrhythmia showed $\mathrm{I}_{\mathrm{Ks}}$ is better able to prevent EADs during increased L-type $\mathrm{Ca}^{2+}$ current.

These studies illustrate how dynamic clamp can be used to experimentally validate computational models, which are typically built from heterogenous data sets spanning numerous experiments, under consistent conditions. Thereafter, new data can be used to further refine the models and advance mechanistic understanding.

\section{DRUG SAFETY TESTING PLATFORMS}

Dynamic clamp has also been utilized in the development of new assays for assessment of drug proarrhythmic risks. The current regulatory framework used to prevent approval of drugs with the potential to induce TdP is focused on two main areas: the propensity of the drug to block the hERG channel in vitro, and whether the drug prolongs the QTc interval of the ECG. Though largely successful at preventing proarrhythmic drugs from entering the market, the approach has been criticized due to its low specificity, as hERG block and QT prolongation do not always carry torsadogenic risk (Sager et al., 2014; Colatsky et al., 2016). Consequently, it is 
generally agreed that many promising drugs that may have little arrhythmogenic risk have their development terminated due to failing either criteria. As mentioned previously, the CiPA initiative considers human stem-cell derived cardiomyocytes a key component in future drug safety assays (Sager et al., 2014; Colatsky et al., 2016), and dynamic clamp has been used to address key limitations.

Human induced pluripotent stem cell derived cardiomyocytes (hiPSC-CMs) are being used as an alternative to traditional animal models, cell lines, and heterologous expression systems in the study of cardiac electrophysiology mechanisms and druginduced arrhythmia. Due to the inherent difficulty in obtaining human cardiac tissue for study, hiPSC-CMs may provide an accessible source of human cell lines and includes the additional capacity to produce patient-specific lines. However, as with human embryonic stem cell derived cardiomyocytes, hiPSC-CMs exhibit an immature phenotype. These cells are stereotypically characterized by spontaneous activity, elevated maximum diastolic potentials, low maximum upstroke velocity, and highly variable APD (Hoekstra et al., 2012). A major contributing factor for these issues is hiPSC-CMs lack of the inward rectifying $\mathrm{K}^{+}$current $\left(\mathrm{I}_{\mathrm{K} 1}\right)$, which plays a major role maintaining a stable resting potential in quiescent cardiomyocytes (Doss et al., 2012). The lack of $I_{K 1}$ is a cumulative issue, in that a generally depolarized membrane potential influences other cardiac currents, such as lowering the availability of fast $\mathrm{Na}^{+}$channels due to inactivation, which reduces upstroke velocity.

Bett et al. implemented a dynamic clamp based approach to resolve the immaturity issue in hiPSC-CMs through the addition of a virtual $\mathrm{I}_{\mathrm{K} 1}$ current (Bett et al., 2013). The original erratic AP morphology of hiPSC-CMs (Figure 2A) was transformed to an AP profile similar to those seen in adult human cardiomyocytes (Figure 2B), with a stable resting membrane potential and fast upstroke velocity. Seeking to test the impact of the dynamic clamp transformation in response to drug perturbation, hiPSCCMs were exposed to the $\mathrm{Ca}^{2+}$ agonist BayK-8644 at room temperature. Without dynamic clamp, drug addition ceased spontaneous AP generation (Figure 2C) most likely due to BayK8644 induced $\mathrm{Ca}^{2+}$ loading. This is in stark contrast to what is expected from ventricular cardiomyocytes in humans and other mammalian species, where an increase in depolarizing $\mathrm{Ca}^{2+}$ currents is expected to increase APD and abnormal activity, such as EADs. With $\mathrm{I}_{\mathrm{K} 1}$ dynamic clamp, however, APD prolongation is evident in stimulated APs (Figure 2D). This illustrates that while hiPSC-CMs are sensitive to BayK-8644, lack of $\mathrm{I}_{\mathrm{K} 1}$ can mask the relevance of drug effects.

Building upon this work, Putten et al. used multiple $\mathrm{I}_{\mathrm{K} 1}$ models in their dynamic clamp experiments to examine the impact of varying degrees of rectification (Meijer van Putten et al., 2015), a biological feature of the $\mathrm{I}_{\mathrm{K} 1}$ current due to differential expression of the channel (Kir2.x) subunits (Wang et al., 1998). Additionally, $\mathrm{I}_{\mathrm{K} 1}$ channelopathies were investigated by modifying their Kir2.1 model to represent gain-of-function and loss-of-function mutations. The gain-of-function mutation was based on the E299V mutation associated with short QT syndrome 3 , and the loss-of-function mutation was based on the heterozygous dominant-negative mutation in KCNJ2 associated with Andersen-Tawil syndrome. The top panel of Figure $2 \mathrm{E}$ plots the different current-voltage relationships of the modified models. The bottom panel of Figure $2 \mathrm{E}$ shows the corresponding APs when these models are used in the calculation of the virtual $\mathrm{I}_{\mathrm{K} 1}$ current during dynamic clamp. Consistent with short QT, the gain-of-function mutation significantly decreased APD, while the loss-of-function had only a marginal effect.

More recently, hiPSC-CM studies augmented with $\mathrm{I}_{\mathrm{K} 1}$ dynamic clamp have provided insight into cardiac abnormalities such as Brugada syndrome (Veerman et al., 2016), long QT syndrome (Rocchetti et al., 2017), and familial atrial fibrilliation (Marczenke et al., 2017). While ion channel dysfunction has been associated with Brugada Syndrome, mainly the cardiac fast $\mathrm{Na}^{+}$current, Veerman et al. found no clear cellular electrophysiological abnormalities in patient-derived hiPSCCMs, suggesting that other factors, such as fibrosis, could also be underlying mechanisms (Veerman et al., 2016). Rocchetti et al. recently studied hiPSC-CMs derived from a long QT patient carrying a heterozygous mutation in one of the three calmodulin encoding genes (Rocchetti et al., 2017). The patient-specific cells exhibited prolonged APD and failure to shorten with increased pacing rate, which the study linked to impairment of $\mathrm{Ca}^{2+}$-dependent inactivation of $\mathrm{I}_{\mathrm{CaL}}$. The $\mathrm{I}_{\mathrm{CaL}}$ blocker verapamil reversed mutation-induced repolarization abnormalities. Marczenke et al. explored the role of mutations of the KCNA5 gene, encoding the channel responsible for the ultrarapid delayed rectifier $\mathrm{K}^{+}$current, in familial atrial fibrillation (Marczenke et al., 2017). The authors generated a functional KCNA5 knockout hiPSC-CM line combining CRISPR/Cas9-mediated mutagenesis and atrial- or ventricularspecific differentiation through manipulation of retinoic acid signaling (Devalla et al., 2015). They observed a strictly atrialspecific disease phenotype, where atrial KCNA5 knockout hiPSCCMs exhibited prolonged APD and EADs at low stimulation frequencies vs. insignificant changes in the ventricular variant. These works highlight the potential of hiPSC-CMs in cardiac patient-specific and subtype-specific disease modeling.

$\mathrm{I}_{\mathrm{K} 1}$ dynamic clamp is becoming more common to hiPSC-CM studies to reduce variability in experimental metrics, eliminate spontaneity due to elevated resting membrane potential, and yield a more physiological relevant phenotype. Verkerk et al. systematically analyzed the impact of $\mathrm{I}_{\mathrm{K} 1}$ dynamic clamp on AP characteristics in atrial and ventricular hiPSC-CMs, and provided an in-depth comparison of the methodology and experimental variability of the studies discussed above (Verkerk et al., 2017). While $\mathrm{I}_{\mathrm{K} 1}$ dynamic clamp appears to reduce the variability of most $\mathrm{AP}$ parameters, enthusiasm of reducing the large experimental variability of hiPSC-CMs is tempered by the observation that APD variability is not affected. However, elimination of spontaneous depolarizations allows for stimulus at static frequencies, permitting investigation into rate-dependence. More importantly, static pacing reduces beat-to-beat variability, granting a greater ability to detect AP parameter changes. Verkerk et al. also investigated the impact of different mathematical formulations of the injected 
A

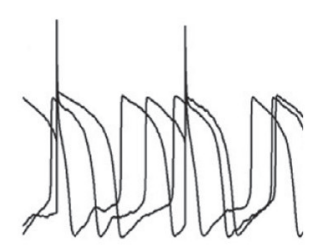

B
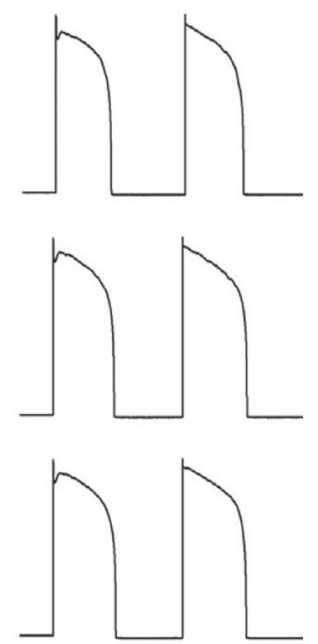

C
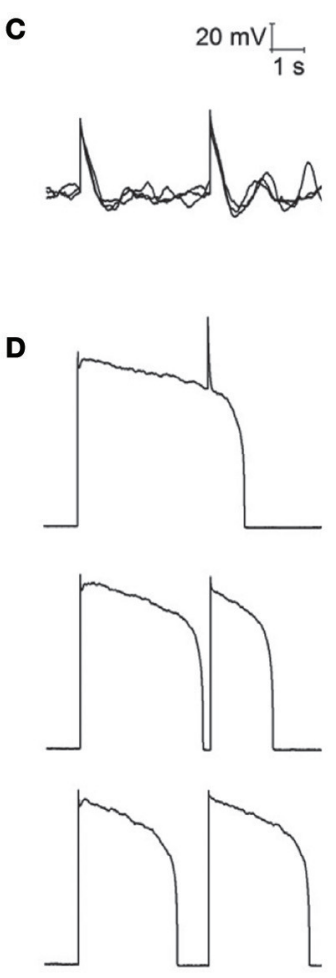

E
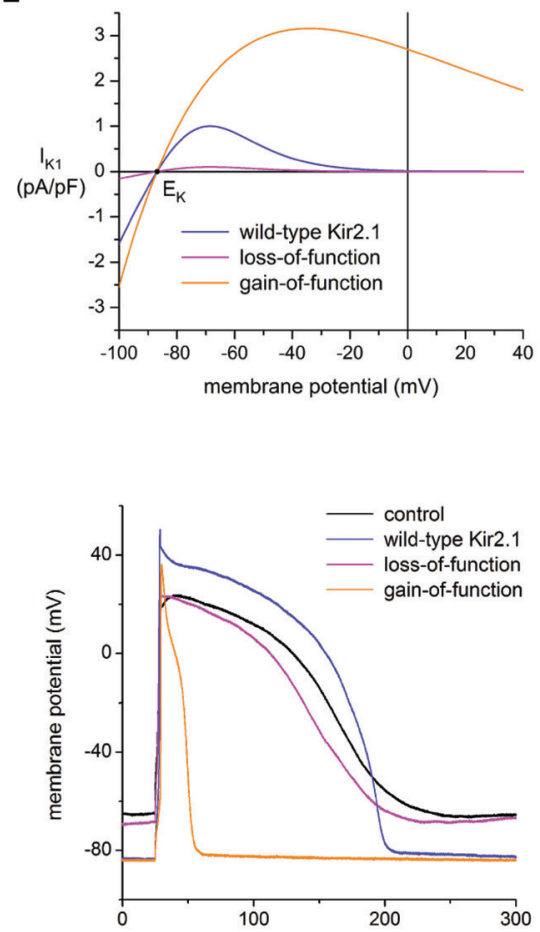

FIGURE 2 | Addressing the immature electrophysiological phenotype of hiPSC-CMs. General lack of the $\mathrm{I}_{\mathrm{K} 1} \mathrm{Current}$ in hiPSC-CMs plays a major role in their immature phenotype, which was compensated for through $I_{\mathrm{K} 1}$ dynamic clamp. (A) Spontaneous and erratic activity is typical of hiPSC-CMs (average resting potential $=-63 \pm$ $5.8 \mathrm{mV}, n=21$ ). (B) After injection of a virtual $I_{K 1}$ current via dynamic clamp, cells become quiescent and produce adult-like stimulated APs (average resting potential $=-84 \pm 0.1, n=21)$. (C) When exposed to the $\mathrm{Ca}^{2+}$ agonist BayK-8644, increased $\mathrm{Ca}^{2+}$ loading terminated spontaneous AP generation. (D) Exposure of BayK-8644 along with $I_{K 1}$ dynamic clamp prolonged APD compared to (B). (A-D) adapted with permission (Bett et al., 2013). (E) Top panel, current to voltage relationships of $\mathrm{I}_{\mathrm{K} 1}$ models representing wild-type Kir2.1, loss-of-function mutation, and gain-of-function mutation. Corresponding AP morphology during dynamic clamp injection of hiPSC-CMs of each model is shown in the bottom panel. Adapted with permission (Meijer van Putten et al., 2015).

$\mathrm{I}_{\mathrm{K} 1}$ current, by comparing the models used in several studies discussed previously (Bett et al., 2013; Meijer van Putten et al., 2015; Rocchetti et al., 2017). Not surprisingly, the parameter selection of $\mathrm{I}_{\mathrm{K} 1}$ current density and kinetics can influence relevant AP metrics. Conversely, the flexibility inherent to model modification provides a means to tailor the $\mathrm{I}_{\mathrm{K} 1}$ current to specific cell types, such as ventricular or atrial.

The low throughput of dynamic clamp is a major limitation to its use as part of a drug testing hiPSC-CM platform. Techniques to increase maturation and $\mathrm{I}_{\mathrm{K} 1}$ density, such as $3 \mathrm{D}$ culturing (Lemoine et al., 2017) and adenovirus-mediated overexpression of $\mathrm{I}_{\mathrm{K} 1}$ (Vaidyanathan et al., 2016), may circumvent the need for dynamic clamp, but are currently not widely used. Automated patch clamp offers a possible route to increase throughput, but brings a new set of issues, such as interfacing with proprietary equipment and the use of single suspended cells. In a promising recent advance, Goversen et al. have successfully combined $\mathrm{I}_{\mathrm{K} 1}$ dynamic clamp with automated patch clamp of hiPSC-CMs, suggesting the feasibility of high-throughput application as a drug testing platform (Goversen et al., 2017).

In summary, dynamic clamp has been utilized in a number of exciting studies to address some of the inherent limitations of hiPSC-CMs, suggesting a promise as a component of safety pharmacology testing. Furthermore, the ability to modify the underlying mathematical models to examine channelopathies expands the capabilities of this platform.

\section{CONCLUSION}

By coupling mathematical models with biological experiments, dynamic clamp has provided a powerful tool in the search for potential anti-arrhythmic therapies through modelbased perturbations, enhanced hiPSC-CMs as a platform for pharmacological safety testing, and used to clarify and improve mathematical models of cardiac electrophysiology. Dynamic clamp allows fine manipulation of numerous parameters like in-silico studies, but is performed in the context of experimental biology. This approach has enabled investigators to test theoretical perturbations in real-time and in live cells, and the power of this technique is represented by the broadness seen in the studies discussed here. It is expected dynamic clamp will continue to elucidate the mechanisms underlying cardiac arrhythmia and identify novel drug targets, and could evolve into a high-throughput assay, e.g., on automated patch clamp platforms to improve maturity of hiPSC-CMs. 


\section{AUTHOR CONTRIBUTIONS}

FO, EG, TK-M, and DC all contributed to the planning, writing, and editing of the manuscript and figures contained herein.

\section{REFERENCES}

Ahrens-Nicklas, R. C., and Christini, D. J. (2009). Anthropomorphizing the mouse cardiac action potential via a novel dynamic clamp method. Biophys. J. 97, 2684-2692. doi: 10.1016/j.bpj.2009.09.002

Altomare, C., Bartolucci, C., Sala, L., Bernardi, J., Mostacciuolo, G., Rocchetti, M., et al. (2015). IKr impact on repolarization and its variability assessed by dynamic clamp. Circ. Arrhythm. Electrophysiol. 8, 1265-1275. doi: 10.1161/CIRCEP.114.002572

Antzelevitch, C., Sicouri, S., Litovsky, S. H., Lukas, A., Krishnan, S. C., Diego, J. M. D., et al. (1991). Heterogeneity within the ventricular wall. Electrophysiology and pharmacology of epicardial, endocardial, and $M$ cells. Circ. Res. 69, 1427-1449. doi: 10.1161/01.RES.69.6.1427

Aréchiga-Figueroa, I. A., Rodríguez-Martínez, M., Albarado, A., Torres-Jácome, J., and Sánchez-Chapula, J. A. (2010). Multiple effects of 4-aminopyridine on feline and rabbit sinoatrial node myocytes and multicellular preparations. Pflugers Arch. 459, 345-355. doi: 10.1007/s00424-009-0734-3

Bartolucci, C., Altomare, C., Bennati, M., Furini, S., Zaza, A., and Severi, S. (2015). Combined action potential- and dynamic-clamp for accurate computational modelling of the cardiac $\mathrm{I}_{\mathrm{Kr}}$ current. J. Mol. Cell. Cardiol. 79, 187-194. doi: 10.1016/j.yjmcc.2014.11.011

Berecki, G., Zegers, J. G., Bhuiyan, Z. A., Verkerk, A. O., Wilders, R., and Van Ginneken, A. C. G. (2006). Long-QT syndrome-related sodium channel mutations probed by the dynamic action potential clamp technique. J. Physiol. 570, 237-250. doi: 10.1113/jphysiol.2005.096578

Berecki, G., Zegers, J. G., Verkerk, A. O., Bhuiyan, Z. A., de Jonge, B., Veldkamp, M. W., et al. (2005). HERG channel (dys)function revealed by dynamic action potential clamp technique. Biophys. J. 88, 566-578. doi: 10.1529/biophys. 104.047290

Bett, G. C., Kaplan, A. D., Lis, A., Cimato, T. R., Tzanakakis, E. S., Zhou, Q., et al. (2013). Electronic "expression" of the inward rectifier in cardiocytes derived from human-induced pluripotent stem cells. Heart Rhythm 10, 1903-1910. doi: 10.1016/j.hrthm.2013.09.061

Bettencourt, J. C., Lillis, K. P., Stupin, L. R., and White, J. A. (2008). Effects of imperfect dynamic clamp: computational and experimental results. J. Neurosci. Methods 169, 282-289. doi: 10.1016/j.jneumeth.2007.10.009

Bot, C. T., Kherlopian, A. R., Ortega, F. A., Christini, D. J., and Krogh-Madsen, T. (2012). Rapid genetic algorithm optimization of a mouse computational model: benefits for anthropomorphization of neonatal mouse cardiomyocytes. Comput. Physiol. Med. 3:421. doi: 10.3389/fphys.2012.00421

Brown, T. R., Krogh-Madsen, T., and Christini, D. J. (2016). Illuminating myocytefibroblast homotypic and heterotypic gap junction dynamics using dynamic clamp. Biophys. J. 111, 785-797. doi: 10.1016/j.bpj.2016.06.042

Colatsky, T., Fermini, B., Gintant, G., Pierson, J. B., Sager, P., Sekino, Y., et al. (2016). The comprehensive in vitro Proarrhythmia Assay (CiPA) initiative - Update on progress. J. Pharmacol. Toxicol. Methods 81, 15-20. doi: 10.1016/j.vascn.2016.06.002

Cranefield, P. F., and Aronson, R. S. (1991). Torsades de pointes and early afterdepolarizations. Cardiovasc. Drugs Ther. 5, 531-537. doi: 10.1007/BF03029780

Devalla, H. D., Schwach, V., Ford, J. W., Milnes, J. T., El-Haou, S., Jackson, C., et al. (2015). Atrial-like cardiomyocytes from human pluripotent stem cells are a robust preclinical model for assessing atrial-selective pharmacology. EMBO Mol. Med. 7, 394-410. doi: 10.15252/emmm.201404757

Devenyi, R. A., Ortega, F. A., Groenendaal, W., Krogh-Madsen, T., Christini, D. J., and Sobie, E. A. (2017). Differential roles of two delayed rectifier potassium currents in regulation of ventricular action potential duration and arrhythmia susceptibility. J. Physiol. 595, 2301-2317. doi: 10.1113/JP273191

\section{FUNDING}

This work was funded by NIH grants U01HL136297 (to DC) and R01HL131517 (to EG), and the American Heart Association (15SDG24910015 to EG).

Dong, M., Sun, X., Prinz, A. A., and Wang, H.-S. (2006). Effect of simulated Ito on guinea pig and canine ventricular action potential morphology. Am. J. Physiol. Heart Circ. Physiol. 291, H631-H637. doi: 10.1152/ajpheart.00084.2006

Dong, M., Yan, S., Chen, Y., Niklewski, P. J., Sun, X., Chenault, K., et al. (2010). Role of the transient outward current in regulating mechanical properties of canine ventricular myocytes. J. Cardiovasc. Electrophysiol. 21, 697-703. doi: 10.1111/j.1540-8167.2009.01708.x

Doss, M. X., Diego, J. M. D., Goodrow, R. J., Wu, Y., Cordeiro, J. M., Nesterenko, V. V., et al. (2012). Maximum diastolic potential of human induced pluripotent stem cell-derived cardiomyocytes depends critically on IKr. PLoS ONE 7:e40288. doi: 10.1371/journal.pone.0040288

Goversen, B., Becker, N., Stoelzle-Feix, S., Obergrussberger, A., van Veen, T., Fertig, N., et al. (2017). A hybrid model for safety pharmacology on an automated patch clamp platform: using dynamic clamp to join iPSC-derived cardiomyocytes and simulations of $\mathrm{I}_{\mathrm{K} 1}$ ion channels in real-time. Front. Physiol. 8:1094. doi: 10.3389/fphys.2017.01094

Hoekstra, M., Mummery, C. L., Wilde, A. A. M., Bezzina, C. R., and Verkerk, A. O. (2012). Induced pluripotent stem cell derived cardiomyocytes as models for cardiac arrhythmias. Front. Physiol. 3:346. doi: 10.3389/fphys.2012.00346

Huelsing, D. J., Pollard, A. E., and Spitzer, K. W. (2001). Transient outward current modulates discontinuous conduction in rabbit ventricular cell pairs. Cardiovasc. Res. 49, 779-789. doi: 10.1016/S0008-6363(00)00300-X

January, C. T., and Riddle, J. M. (1989). Early afterdepolarizations: mechanism of induction and block. A role for L-type $\mathrm{Ca}^{2+}$ current. Circ. Res. 64, 977-990. doi: 10.1161/01.RES.64.5.977

Joyner, R. W., Sugiura, H., and Tan, R. C. (1991). Unidirectional block between isolated rabbit ventricular cells coupled by a variable resistance. Biophys. J. 60, 1038-1045. doi: 10.1016/S0006-3495(91)82141-5

Karagueuzian, H. S., Pezhouman, A., Angelini, M., and Olcese, R. (2017). Enhanced late $\mathrm{Na}$ and $\mathrm{Ca}$ currents as effective antiarrhythmic drug targets. Front. Pharmacol. 8:36. doi: 10.3389/fphar.2017.00036

Lemoine, M. D., Mannhardt, I., Breckwoldt, K., Prondzynski, M., Flenner, F., Ulmer, B., et al. (2017). Human iPSC-derived cardiomyocytes cultured in 3D engineered heart tissue show physiological upstroke velocity and sodium current density. Sci. Rep. 7:5464. doi: 10.1038/s41598-017-05600-w

Luo, C. H., and Rudy, Y. (1994). A dynamic model of the cardiac ventricular action potential. I. Simulations of ionic currents and concentration changes. Circ. Res. 74, 1071-1096. doi: 10.1161/01.RES.74.6.1071

Madhvani, R. V., Angelini, M., Xie, Y., Pantazis, A., Suriany, S., Borgstrom, N. P., et al. (2015). Targeting the late component of the cardiac L-type $\mathrm{Ca}^{2+}$ current to suppress early afterdepolarizations. J. Gen. Physiol. 145, 395-404. doi: 10.1085/jgp.201411288

Madhvani, R. V., Xie, Y., Pantazis, A., Garfinkel, A., Qu, Z., Weiss, J. N., et al. (2011). Shaping a new $\mathrm{Ca}^{2+}$ conductance to suppress early afterdepolarizations in cardiac myocytes. J. Physiol. 589, 6081-6092. doi: 10.1113/jphysiol.2011.219600

Marczenke, M., Piccini, I., Mengarelli, I., Fell, J., Röpke, A., Seebohm, G., et al. (2017). Cardiac subtype-specific modeling of $\mathrm{K}_{v} 1.5$ ion channel deficiency using human pluripotent stem cells. Front. Physiol. 8:469. doi: 10.3389/fphys.2017.00469

McSpadden, L. C., Nguyen, H., and Bursac, N. (2012). Size and ionic currents of unexcitable cells coupled to cardiomyocytes distinctly modulate cardiac action potential shape and pacemaking activity in micropatterned cell pairs. Circ. Arrhythm. Electrophysiol. 5, 821-830. doi: 10.1161/CIRCEP.111.969329

Meijer van Putten, R. M., Mengarelli, I., Guan, K., Zegers, J. G., Ginneken, V., et al. (2015). Ion channelopathies in human induced pluripotent stem cell derived cardiomyocytes: a dynamic clamp study with virtual IK1. Front. Physiol. 6:7. doi: 10.3389/fphys.2015.00007 
Nguyen, T. P., Singh, N., Xie, Y., Qu, Z., and Weiss, J. N. (2015). Repolarization reserve evolves dynamically during the cardiac action potential: effects of transient outward currents on early afterdepolarizations. Circ. Arrhythm. Electrophysiol. 8, 694-702. doi: 10.1161/CIRCEP.114.002451

Nguyen, T. P., Xie, Y., Garfinkel, A., Qu, Z., and Weiss, J. N. (2012). Arrhythmogenic consequences of myofibroblast-myocyte coupling. Cardiovasc. Res. 93, 242-251. doi: 10.1093/cvr/cvr292

Ortega, F. A., Butera, R. J., Christini, D. J., White, J. A., and Dorval, A. D. (2014). "Dynamic clamp in cardiac and neuronal systems using RTXI," in Patch-Clamp Methods and Protocols Methods in Molecular Biology, (New York, NY: Humana Press), 327-354.

Patel, Y. A., George, A., Dorval, A. D., White, J. A., Christini, D. J., and Butera, R. J. (2017). Hard real-time closed-loop electrophysiology with the Real-Time eXperiment Interface (RTXI). PLoS Comput. Biol. 13:e1005430. doi: 10.1371/journal.pcbi.1005430

Prinz, A. A., Abbott, L. F., and Marder, E. (2004). The dynamic clamp comes of age. Trends Neurosci. 27, 218-224. doi: 10.1016/j.tins.2004.02.004

Ravagli, E., Bucchi, A., Bartolucci, C., Paina, M., Baruscotti, M., DiFrancesco, D., et al. (2016). Cell-specific dynamic clamp analysis of the role of funny If current in cardiac pacemaking. Prog. Biophys. Mol. Biol. 120, 50-66. doi: 10.1016/j.pbiomolbio.2015.12.004

Ridley, J. M., Milnes, J. T., Zhang, Y. H., Witchel, H. J., and Hancox, J. C. (2003). Inhibition of HERG K+ current and prolongation of the guineapig ventricular action potential by 4 -aminopyridine. J. Physiol. 549, 667-672. doi: 10.1113/jphysiol.2003.043976

Robinson, H. P., and Kawai, N. (1993). Injection of digitally synthesized synaptic conductance transients to measure the integrative properties of neurons. J. Neurosci. Methods 49, 157-165. doi: 10.1016/0165-0270(93)90119-C

Rocchetti, M., Sala, L., Dreizehnter, L., Crotti, L., Sinnecker, D., Mura, M., et al. (2017). Elucidating arrhythmogenic mechanisms of long-QT syndrome CALM1-F142L mutation in patient-specific induced pluripotent stem cell-derived cardiomyocytes. Cardiovasc. Res. 113, 531-541. doi: $10.1093 / \mathrm{cvr} / \mathrm{cvx} 006$

Roden, D. M. (1998). Taking the "idio" out of "idiosyncratic": predicting torsades de pointes. Pacing Clin. Electrophysiol. 21, 1029-1034. doi: 10.1111/j.1540-8159.1998.tb00148.x

Sager, P. T., Gintant, G., Turner, J. R., Pettit, S., and Stockbridge, N. (2014). Rechanneling the cardiac proarrhythmia safety paradigm: a meeting report from the Cardiac Safety Research Consortium. Am. Heart J. 167, 292-300. doi: 10.1016/j.ahj.2013.11.004

Sanguinetti, M. C., and Jurkiewicz, N. K. (1990). Two components of cardiac delayed rectifier $\mathrm{K}+$ current. Differential sensitivity to block by class III antiarrhythmic agents. J. Gen. Physiol. 96, 195-215. doi: 10.1085/jgp.96.1.195

Sharp, A. A., O’Neil, M. B., Abbott, L. F., and Marder, E. (1993). Dynamic clamp: computer-generated conductances in real neurons. J. Neurophysiol. 69, 992-995.

Spitzer, K. W., Sato, N., Tanaka, H., Firek, L., Zaniboni, M., and Giles, W. R. (1997). Electrotonic modulation of electrical activity in rabbit atrioventricular node myocytes. Am. J. Physiol. 273, H767-H776.
Tan, R. C., and Joyner, R. W. (1990). Electrotonic influences on action potentials from isolated ventricular cells. Circ. Res. 67, 1071-1081. doi: 10.1161/01.RES.67.5.1071

Vaidyanathan, R., Markandeya, Y. S., Kamp, T. J., Makielski, J. C., January, C. T., and Eckhardt, L. L. (2016). I I 1 -enhanced human-induced pluripotent stem cell-derived cardiomyocytes: an improved cardiomyocyte model to investigate inherited arrhythmia syndromes. Am. J. Physiol. Heart Circ. Physiol. 310, H1611-H1621. doi: 10.1152/ajpheart.00481.2015

Verheijck, E. E., Wilders, R., Joyner, R. W., Golod, D. A., Kumar, R., Jongsma, H. J., et al. (1998). Pacemaker synchronization of electrically coupled rabbit sinoatrial node cells. J. Gen. Physiol. 111, 95-112. doi: 10.1085/jgp.111.1.95

Veerman, C. C., Mengarelli, I., Guan, K., Stauske, M., Barc, J., Tan, H. L., et al. (2016). hiPSC-derived cardiomyocytes from Brugada Syndrome patients without identified mutations do not exhibit clear cellular electrophysiological abnormalities. Sci. Rep. 6:30967. doi: 10.1038/srep30967

Verkerk, A. O., Veerman, C. C., Zegers, J. G., Mengarelli, I., Bezzina, C. R., and Wilders, R. (2017). Patch-clamp recording from human induced pluripotent stem cell-derived cardiomyocytes: improving action potential characteristics through dynamic clamp. Int. J. Mol. Sci. 18:1873. doi: 10.3390/ijms 18091873

Wang, Z., Yue, L., White, M., Pelletier, G., and Nattel, S. (1998). Differential distribution of inward rectifier potassium channel transcripts in human atrium versus ventricle. Circulation 98, 2422-2428. doi: 10.1161/01.CIR.98.22.2422

Wilders, R. (2006). Dynamic clamp: a powerful tool in cardiac electrophysiology. J. Physiol. 576, 349-359. doi: 10.1113/jphysiol.2006.115840

Workman, A. J., Marshall, G. E., Rankin, A. C., Smith, G. L., and Dempster, J. (2012). Transient outward K+ current reduction prolongs action potentials and promotes afterdepolarisations: a dynamic-clamp study in human and rabbit cardiac atrial myocytes. J. Physiol. 590, 4289-4305. doi: 10.1113 /jphysiol.2012.235986

Xie, L. H., Chen, F., Karagueuzian, H. S., and Weiss, J. N. (2009). Oxidative stressinduced afterdepolarizations and calmodulin kinase II signaling. Circ. Res. 104, 79-86. doi: 10.1161/CIRCRESAHA.108.183475

Zaniboni, M., Pollard, A. E., Yang, L., and Spitzer, K. W. (2000). Beat-tobeat repolarization variability in ventricular myocytes and its suppression by electrical coupling. Am. J. Physiol. Heart Circ. Physiol. 278, H677-H687. doi: 10.1152/ajpheart.2000.278.3.H677

Conflict of Interest Statement: The authors declare that the research was conducted in the absence of any commercial or financial relationships that could be construed as a potential conflict of interest.

Copyright (c) 2018 Ortega, Grandi, Krogh-Madsen and Christini. This is an openaccess article distributed under the terms of the Creative Commons Attribution License (CC BY). The use, distribution or reproduction in other forums is permitted, provided the original author(s) or licensor are credited and that the original publication in this journal is cited, in accordance with accepted academic practice. No use, distribution or reproduction is permitted which does not comply with these terms. 